\title{
Evaluación de estrategias pedagógicas de implementación del Microdiseño Gurricular de Física en la Facultad de Ingeniería Una experiencia educativa
}

\author{
Álvaro Avendaño R. \\ Profesor Universidad Surcolombiana \\ alave@usco.edu.co \\ Alix Maria Casadiego \\ Profesora Universidad Surcolombianas \\ Alixcasadiego@yahoo.es
}

Resumen

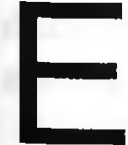
n la Universidad Surcolombiana existe un indice de deserción de $41 \%$, que si bien está por debajo de la media nacional, la propia universidad tiene su cuota de participación en este indice. El presente trabajo de investigación versa sobre el impacto de una propuesta metodológica, no autoritaria y carente de verticalidad, en la disminución de la deserción. La propuesta impulsa la amplia participación de los estudiantes en un proceso en el cual la calificación final depende de un continuo monitoreo de los avances de los estudiantes a lo largo del semestre.

Para el logro de los objetivos se llevó a cabo un estudio cuantitativo y longitudinal lo largo de los períodos comprendidos entre el Semestre B del 2009 y el semestre B del 2011 con el fin de establecer la relación entre variables como participación y obtención de logros. La muestra abarcó a los estudiantes de la asignatura mecánica y electromagnetismo de la Facultad de
Ingeniería (sede Neiva). La falta de éxito en los logros es uno de los factores de deserción.

Palabras clave: deserción, participación, rendimiento, mecánica y electromagnetismo.

\section{Introducción}

El profesor Sánchez $(2009 ; 97)$ en su artículo "Factores de deserción estudiantil en la Universidad Surcolombiana" señala una deserción de $41 \%$ ante una media nacional de $50 \%$. Los autores señalan que de acuerdo al ICFES, las cifras de deserción universitaria, entre los años 1990 y 1999 , oscilaban entre $68,14 \%$ y $47,68 \%$ y, citando a la fundación Restrepo Barco; en el país, el mayor número de estudiantes que desertan lo hace por razones ajenas a ellos y a la institución, los cuales pueden ser pobreza, inequidad, violencia y marginalidad social; como también la relación docente - estudiante y estudiante institución.

Es decir, se señala como causa principal de la deserción el factor socioeconómico, en 
segunda instancia el personal $y$, por último, factores internos de la Universidad, aunque advierten que sobre el segundo factor, la universidad tiene influencia en la situación personal de un estudiante desmotivado e insatisfecho.

El estudio encontró que dentro de los factores de deserción se encuentra la insatisfacción de logros académicos en los cuales influye que no existe una adecuada interacción con el docente produciendo insuficiente retroalimentación en las tareas y actividades que debe el estudiante desarrollar para ser exitoso (Morgan, 2004).

Estos resultados son corroborados por las diferentes acciones que toma el Ministerio de Educación Nacional (2007), cuando propone a las instituciones que presente "Proyectos para disminuir la deserción al $40 \%$ en el 2010 y al $25 \%$ en el 2019

\section{Contexto local, nacional e internacional}

El Ministerio de Educación Nacional hizo esta convocatoria a nivel nacional a las instituciones de educación superior que tuvieran registro en el Sistema Nacional de Información de Educación Superior (SNIES), con la condición de haber registrado el proyecto en el Banco de Experiencias Significativas en Educación Superior y que estuvieran implementado el Sistema de Prevención y Análisis de la Deserción en las Instituciones de Educación Superior (SPADIES). Es decir, aunque amplia, de todas maneras puso sus condiciones limitantes.

La convocatoria formaba parte de la política de "Ampliación de Cobertura en la Educación Superior", la cual implicaba prestar apoyo y fortalecimiento de proyectos y estrategias que se desarrollan para disminuir la deserción estudiantil en educación superior, como una estrategia que reducir la deserción estudiantil.

Disminuir la deserción implica promover ganancias en la competitividad del país dentro del concierto latinoamericano y, por supuesto, ante el gran concierto de naciones de todo el mundo. Todo ello con el fin de obtener un mejor nivel de vida de la población colombiana a través de la educación. La cual, en ese entonces, era considerada como un eje del Plan de Desarrollo del Gobierno. La estrategia cobijaba acciones estratégicas hacia tres objetivos fundamentales: Ampliación de cobertura, incremento de la calidad y la pertinencia, y mejoramiento de la eficacia y eficiencia del sector.

Además, como lo anotaba el Ministerio de Educación Nacional en esa misma convocatoria, no se puede hablar simultáneamente de ampliación de cobertura y de tasas altas de deserción estudiantil. El Ministerio de Educación Nacional, por supuesto, trataba de ser coherente con su política de esos años de ampliación de cobertura, aumentando el número de estudiantes que lograran culminar sus estudios, que no abandonen sus estudios, hecho que según el Ministerio de Educación Nacional, ocurría en primordialmente en los primeros semestres.

Uno de los problemas cardinales al que se ha venido afrontando el plan de desarrollo de educación superior en Colombia atañe a las altos cotas de deserción estudiantil en los niveles de pregrado. Según estadísticas del Ministerio de Educación Nacional de cada 100 estudiantes que ingresan a una institución de educación superior, cerca de la mitad no logra graduarse.

Se señalaba que, para superar esta situación problemática era muy importante averiguar los factores determinantes, no sólo a nivel general y por cada institución, sino también, en lo particular en lo que ocurre con cada estudiante como individuo.

Dentro de este contexto, el presente proyecto aporta conocimiento que sirve para fundamentar y direccionar las acciones, 
proyectos y políticas que se adopten con tal propósito.

El Ministerio de Educación Nacional, en conjunto con las instituciones de educación superior, cuenta con el diseño y la operación de una metodología de seguimiento de la deserción estudiantil llamada "Sistema para la Prevención y Análisis de la Deserción en las Instituciones de Educación Superior (SPADIES)". Esta herramienta permite una visión sectorial del fenómeno, análisis sobre cada una de las variables asociadas a la deserción por cada uno de los estudiantes, así como seguimiento y evaluación a los resultados de las estrategias implementadas para disminuir la deserción.

Con esta metodología el Ministerio de Educación Nacional identificó, en 120 instituciones de educación superior de todo el país, con una muestra de más de 1.100 .000 estudiantes matriculados entre 1998 y 2006 , que los principales determinantes de la deserción estudiantil en pregrado están relacionados con el rendimiento académico previo al ingreso del programa de educación superior, el ingreso familiar y el acceso a al financiamiento y la orientación profesional/vocacional de los estudiantes.

Otro esfuerzo más reciente, realizado por el Ministerio de Educación Nacional es compromiso adquirido por el Ministerio en el "Acuerdo Nacional para Disminuir la Deserción en Educación" de "liderar y coordinar estrategias que apunten a disminuir la deserción estudiantil, a partir de la construcción y fortalecimiento de diferentes acciones orientadas a mejorar las condiciones académicas de los aspirantes y los estudiantes, tales como: nivelación de competencias, acompañamiento académico, articulación con la media, apoyo económico y de bienestar".

Este Acuerdo, fue firmado por representantes de las IES públicas y privadas y el Gobierno y plantea estrategias como financiación, nivelación académica y apoyo vocacional.

Se planeaba otorgar 200 mil nuevos créditos y daremos 144 mil subsidios a los estudiantes; asi mismo, se agregaron nuevas fuentes de financiación sobre la base de la reforma a la Ley 30 , buscando la participación del sector privado. Estos eran los aspectos destacables dentro la política de educación superior encaminada a garantizar el cumplimiento de la política educativa "Educación de calidad, el camino para la prosperidad".

El proyecto arrancaria con inversiones del orden de 800 mil millones de pesos durante los cuatro años del Gobierno Santos. Es de resaltar que, la reforma a la ley 30 tiene un componente importa de Fortalecimiento de la financiación de la educación superior, que definirá nuevas fuentes de financiación, convocando al sector privado como uno de los actores esenciales en este proceso.

El proceso se debe acompañar con procedimientos de nivelación académica por parte de los alumnos: El Ministerio de Educación Nacional, a su vez, tendrá el apuntalamiento por parte de las IES en procesos de nivelación de competencias; se aplicará el sistema para la prevención de la deserción Spadies; se tendrá mayor articulación entre la educación media y la educación superior a través del fortalecimiento del fondo FEM; y se propiciará el acercamiento entre los docentes de educación media y superior; así mismo, se realizarán ajustes normativos para impulsar la flexibilidad curricular y se fortalecerá al docente en áreas pedagógicas de evaluación y manejo de TIC a través del Plan Nacional de formación docente en educación superior.

El Ministerio de Educación Nacional cuenta con el programa "Buscando Carrera", con el cual, ampliándolo en cobertura y con una mejor calidad, intentará promover el apoyo 
vocacional y el bienestar; acompañándolo de una mayor difusión de los resultados del Observatorio Laboral para la Educación. Según el Ministerio de Educación Nacional esto dará soporte al fomento de los diferentes programas de bienestar para estudiantes de mayor vulnerabilidad y fragilidad de nuestra sociedad colombiana.

Todas estas iniciativas y todos estos compromisos rebelan la magnitud inmensa del problema, pues la meta a lograr es reducir del 45 al $40 \%$ la deserción escolar en la educación superior.

La universidad, por su parte, implementó una serie de estímulos a los estudiantes como Becas para postgrados (Consejo superior; 2010) y adoptó el Plan excelencia de la USCO, el cual incluye préstamos Condonables a estudiantes por rendimiento (Consejo Superior; 2005).

A nivel Latinoamericano tenemos una deserción de 50\% (RAMA; 2007) con un total de 15,7 millones de alumnos matriculados en el año 2005.

En chile La deserción se calcula en $53.7 \%$; Argentina, 40\%; Brasil, en las universidades públicas, $74 \%$; México, $53 \%$. Como puede observarse en el siguiente cuadro (MEN, 2007). (Ver gráfico siguiente)

Entre las sugerencias que hace la Universidad Católica de Valparaiso, Chile, es revisión de modelos de enseñanza, expectativas educacionales, resolución de conflictos, motivación y autodeterminación y revisión de prácticas pedagógicas (MEN; 2006). En España, la deserción estudiantil en educación superior, por cohorte, presenta un promedio del $40 \%$

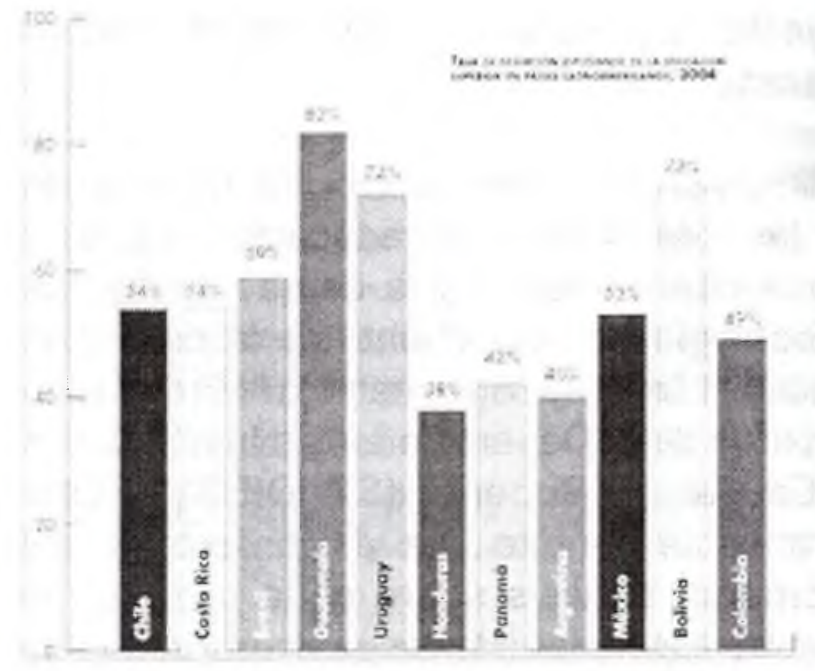

Fuente: Unesco, citado por MEN (2007)

\section{Definiciones y conceptos utilizados}

La deserción se puede definir como el proceso de abandono, voluntario o forzoso de la carrera en la que se matricula un estudiante, por la influencia positiva o negativa de circunstancias internas o externas a él o ella (Universidad de la República de Uruguay; 2003).

Otros autores definen la deserción en la educación superior en forma más operativa como la cantidad de estudiantes que abandona el sistema de educación superior entre uno y otro período académico (semestre - año).La deserción se calcula como el balance entre la matrícula total del primer período, menos los egresados del mismo período y más los estudiantes reintegrados en periodo siguiente, lo cual genera el nuevo estado ideal de alumnos matriculados sin deserción." (ICFES; 1999)

Según la UNESCO (2005), La deserción, entendida como una forma de abandono de los estudios superiores, adopta distintos comportamientos en los estudiantes afectando la continuidad de sus trayectorias escolares. Estos comportamientos se caracterizan por: 
Abandono o suspensión voluntaria y definitiva de los estudios y del sistema de educación superior por parte del alumno o alumna.

Salida de estudiantes debido a deficiencias académicas y consecuentemente bajo rendimiento escolar.

Cambio de carrera (el alumno o alumna continúa en la misma institución pero se incorpora a otra cohorte generacional) o de institución.

Baja de los estudiantes que alteran el orden y la disciplina institucional. Generalmente obstaculiza el ingreso a otra escuela 0 facultad

La deserción se ha analizado desde a lo menos tres perspectivas.

En primer lugar, desde una óptica interna referida a los procesos de enseñanza (calidad educativa, estructura y pertinencia curricular, formación docente, etc.).

Desde una segunda, que pone el énfasis en los procesos externos a lo propiamente educativo (características del estudiantado, situación socioeconómica del país, acceso a financiamiento, etc.).

De una tercera, que se centra en la interacción entre ambos tipos de factores. Para este último enfoque la deserción debe ser considerada como el producto de la interrelación de múltiples factores, que relacionan el sistema educativo y la estructura social y económica de la sociedad; y como una manifestación de los profundos cambios que han acaecido en las instituciones universitarias, en la composición del alumnado y en el mercado de trabajo. Según algunos analistas 12 se pueden identificar tres periodos criticos para la deserción en la trayectoria de los estudiantes:

La transición entre el nivel medio superior y la licenciatura que se caracteriza por el paso de un ambiente conocido a un mundo en apariencia impersonal, lo que implica serios problemas de ajuste para los estudiantes.
El proceso de admisión, cuando el estudiante se forma expectativas equivocadas sobre las instituciones y las condiciones de la vida estudiantil, que al no satisfacerle, pueden conducir a decepciones tempranas y, por consiguiente, a la deserción.

Cuando el estudiante no logra un adecuado rendimiento académico en las asignaturas del plan de estudios y la institución no le proporciona las herramientas necesarias para superar las deficiencias académicas.

La repitencia y la deserción son fenómenos que en muchos casos están concatenados, ya que la investigación demuestra que la repitencia reiterada conduce, por lo general, al abandono de los estudios.

- Tanto la repitencia como la deserción son siempre procesos individuales, si bien pueden constituirse en un fenómeno colectivo o incluso masivo, y ser estudiado como tal. En dicho caso, por lo general, se asocia a la eficiencia del sistema.

Según Tinto (1992) existen tres periodos esenciales en la aparición del fenómeno de la deserción:

Primer periodo crítico: Se presenta en la transición entre el nivel medio superior y la licenciatura, y se caracteriza por el paso de un ambiente conocido a un mundo en apariencia impersonal, lo que implica serios problemas de ajuste para los estudiantes.

Segundo periodo crítico: Ocurre durante el proceso de admisión, cuando el estudiante se forma expectativas equivocadas sobre las instituciones y las condiciones de la vida estudiantil, que al no satisfacerse, pueden conducir a decepciones tempranas $y$, por consiguiente, a la deserción.

- Tercer periodo crítico: Se origina cuando el estudiante no logra un adecuado rendimiento académico en las asignaturas del plan de estudios y la institución no le proporciona las herramientas necesarias para superar las deficiencias académicas. 
La permanencia del estudiante en el plan de estudios de la licenciatura también se encuentra afectada por el rezago escolar, el cual consiste en el retraso en la inscripción a las asignaturas subsecuentes del plan de estudios en un lapso regular $u$ ordinario.

La motivación y la participación activa del alumnado son elementos fundamentales de los procesos de aprendizaje y una importante preocupación para buena parte del profesorado. ¿Cómo se puede conseguir una mayor implicación de los estudiantes? ¿Cuáles son las estrategias participativas y reflexivas que facilitan esta implicación? ¿Cuáles son las condiciones necesarias para la realización de experiencias y buenas prácticas de participación? ¿Con qué dificultades tenemos que contar y cómo las podemos superar? Los autores abordan estas cuestiones desde el modelo pedagógico del aprendizaje reflexivo experimental. Un modelo, como dicen, poco conocido en el campo de la educación superior, a pesar de su enorme potencial para la formación

Y la mejora de la práctica docente y que estamos experimentando, con buenos resultados, en algunos de los talleres de formación del profesorado universitario.

En cuanto a la participación (MUÑOZ;2008), contribuye a la búsqueda de nuevas vías de acceso al desarrollo cooperativo de un pensamiento científico elaborado desde la autonomía de los sujetos y, al mismo tiempo, como una aventura solidaria, más allá de cualquier forma de voluntarismo.

El término participación, involucra la pretensión de analizar y buscar alternativas a la transmisión del conocimiento que se origina en las aulas de la universidad, entendida como pura transmisión unidireccional (o metodología del busto parlante), en que se realiza una disertación de un tema por parte del profesorado delante de un alumnado que escucha pasivamente y que, como máximo, toma notas o apuntes e interviene, ocasionalmente, cuando es interpelado a hacerlo.

Hay, como mínimo, dos maneras enseñar el conocimiento académico: mediante el aprendizaje pasivo del alumnado (denominado pasivo porque el protagonismo lo asume el docente mediante la sesión de transmisión) y el aprendizaje activo, en el que el alumnado asume más protagonismo en su participación en la enseñanza. Este último aprendizaje también se puede denominar, con matices o cuando se introduzcan ciertos elementos en la participación, interactivo y cooperativo.

Estos últimos pretenden que el alumnado se implique en el proceso de enseñanzaaprendizaje para consolidarlo y significarlo más. Estas dos maneras de transmitir conocimientos tienen lugar en la universidad, aunque podemos encontrar una multitud de matices (como un campo de conocimiento en que intervienen diferentes variables). Pero que el alumnado participe no quita protagonismo al docente, ya que el profesorado tiene un papel importante en tanto que diseñador de espacios de aprendizaje y como guía del proceso de enseñanza-aprendizaje.

\section{Estimular la mayor palpitación en clase.}

Recogiendo las recomendaciones del estudio y respondiendo al llamado del Ministerio de Educación, la presente investigación se unió a ese gran esfuerzo conjunto que hacemos los colombianos por mantener con vida las Universidades Estatales, implementado una estrategia pedagógica en la que exista una mayor comunicación estudiante - docente que impulse la participación de los estudiantes en el aula de clase.

Básicamente se diseñó un proceso continuo de evaluación en el cual se asignan puntos por participación. Cada punto tiene un valor 
adicional para la nota definitiva del estudiante. No son obligatorios y si un estudiante no participa no hay detrimento de su nota acorde con los pactos y compromisos adoptados al comienzo del semestre.

\section{La asignación de puntos tiene varias} modalidades:

Cada vez que se explicaba algo, se les preguntaba, tratando de averiguar la interpretación que ellos hacen de lo expuesto. Si coincide con lo que esperamos los académicos se les otorga puntos.

También se otorgaba puntos por dar opiniones sobre lo afirmado por el profesor o por otros compañeros

Por resolver problemas

Por participar en talleres

Por hacer preguntas pertinentes

Por advertir errores no voluntarios en lo expuesto por el profesor o compañero.

Por cada participación se otorga un punto.

\section{Resultados}

En total se acumularon 161 puntos, se encontró que los puntos, expresados en porcentaje, se habian distribuido como se ilustra en la TABLA 1. Distribución porcentual de puntos

La TABLA 2, se construye a partir de la TABLA 1, pero ahora, acumulando el porcentaje de participación:

Se observa que los que tenían nota superiores a 4.5 obtuvieron el $51 \%$ de los puntos mientras que, aquellos que tenían nota inferior a 2,0 , apenas el $20 \%$ del total de los puntos.

Este es un resultado sorprendente, porque se espera que los estudiantes que tienen bajas notas, sean los que más se esfuercen por
TABLA 1. Distribución porcentual de puntos

\begin{tabular}{|l|l|l|l|}
\hline $\begin{array}{l}\text { Calificació } \\
n\end{array}$ & $\begin{array}{l}\text { Participació } \\
n\end{array}$ & $\begin{array}{l}\text { Calificació } \\
n\end{array}$ & Participación \\
\hline 10 & 1 & 28 & 0 \\
\hline 10 & 2 & 28 & 7 \\
\hline 10 & 2 & 30 & 1 \\
\hline 10 & 4 & 30 & 5 \\
\hline 10 & 5 & 32 & 1 \\
\hline 13 & 1 & 35 & 0 \\
\hline 15 & 1 & 38 & 8 \\
\hline 15 & 3 & 45 & 1 \\
\hline 18 & 0 & 45 & 5 \\
\hline 18 & 1 & 45 & 7 \\
\hline 20 & 5 & 45 & 9 \\
\hline 23 & 4 & 46 & 9 \\
\hline 25 & 0 & 50 & 8 \\
\hline 27 & 1 & 50 & 11 \\
\hline
\end{tabular}

TABLA 2. Porcentaje acumulado de participación

\begin{tabular}{|c|c|c|c|}
\hline $\begin{array}{l}\text { Porcentaje } \\
\text { participación }\end{array}$ & de Calificación & $\begin{array}{l}\text { Porcentaje } \\
\text { participación }\end{array}$ & de Calificación \\
\hline 1 & 10 & 35 & 28 \\
\hline 2 & 10 & 35 & 28 \\
\hline 4 & 10 & 36 & 30 \\
\hline 8 & 10 & 42 & 30 \\
\hline 13 & 10 & 42 & 32 \\
\hline 14 & 13 & 42 & 35 \\
\hline 15 & 15 & 50 & 38 \\
\hline 18 & 15 & 51 & 45 \\
\hline 18 & 18 & 55 & 45 \\
\hline 19 & 18 & 62 & 45 \\
\hline 24 & 20 & 72 & 45 \\
\hline 28 & 23 & 81 & 46 \\
\hline 28 & 25 & 89 & 50 \\
\hline 28 & 27 & 100 & 50 \\
\hline
\end{tabular}


Los anteriores datos se representan en el GRAFICO 1.:

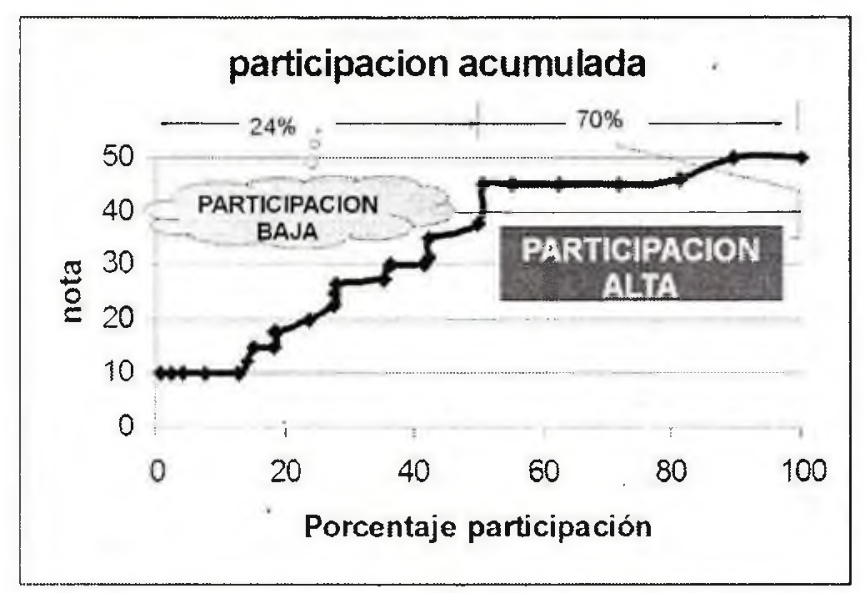

GRAFICO 1. Participación y calificación.

participar. Surge entonces la pregunta: ¿por qué los estudiantes de bajo rendimiento se niegan a parțicipar?

En la TABLA 3, se registra la asistencia y la calificación obtenida en los exámenes:

TABLA 3. Porcentaje de asistencia y rendimiento:

\begin{tabular}{|c|c|c|c|}
\hline $\begin{array}{l}\text { Porcentaje } \\
\text { de } \\
\text { asistencia }\end{array}$ & Calificación & $\begin{array}{l}\text { Porcentaje } \\
\text { de } \\
\text { asistencia }\end{array}$ & Calificación \\
\hline 26 & 18 & 95 & 20 \\
\hline 47 & 13 & 95 & 25 \\
\hline 58 & 10 & 95 & 38 \\
\hline 58 & 10 & 95 & 50 \\
\hline 68 & 35 & 95 & 50 \\
\hline 68 & 45 & 100 & 15 \\
\hline 74 & 27 & 100 & 23 \\
\hline 79 & 10 & 100 & 28 \\
\hline 79 & 30 & 100 & 28 \\
\hline 84 & 10 & 100 & 30 \\
\hline 84 & 32 & 100 & 45 \\
\hline 89 & 15 & 100 & 45 \\
\hline 95 & 10 & 100 & 45 \\
\hline 95 & 18 & 100 & 46 \\
\hline
\end{tabular}

A partir de la TABLA 3, se construye el gráfico 2.

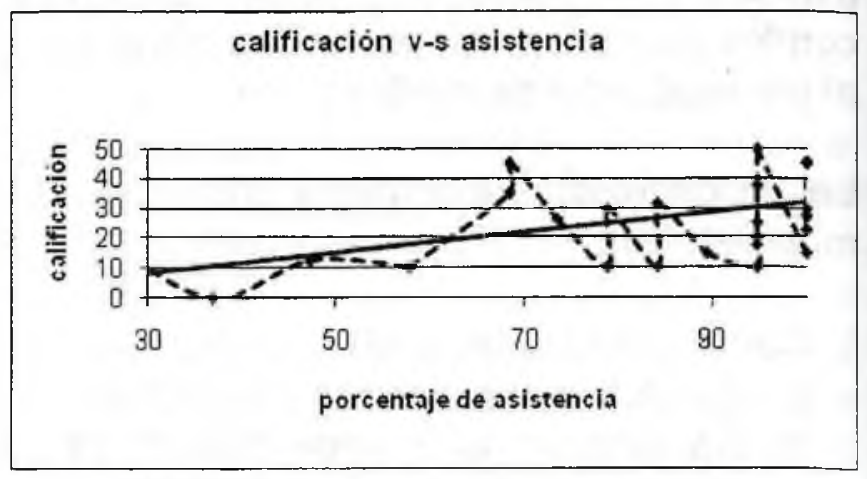

GRAFICO 2. Porcentaje de asistencia y calificación

En él, se resaltan dos aspectos:

Aunque la línea de tendencia (línea sólida) muestra que existe un aumento de la calificación obtenida a medida que se asiste más a clase, de todas maneras los datos son muy dispersos a lado y lado de esta línea $y$, por lo tanto, no se puede afirmar que exista correlación entre estas dos variables.

Se observa que todos los estudiantes, con asistencia menor al $70 \%$, obtienen resultados menores al mínimo de 30 sobre 50 , pero, al mismo tiempo, la simple asistencia no garantiza aprobar la asignatura, pues aún estudiantes con asistencia del $90 \%$ no obtienen resultados mayores a 30 .

En la tabla 3, también puede observarse que el primer cuartil, que corresponde al $25 \%$ de los estudiantes, tiene asistencia menor al $80 \%$. Según la reglamentación vigente, estos estudiantes perderían por fallas. Esto quiere decir que el $25 \%$ del total de los estudiantes, perdería por fallas. En este grupo de baja asistencia, tiene nota aprobatoria el $25 \%$, mientras que, en el resto del grupo, el porcentaje de estudiantes que aprueban es casi el doble: $42 \%$. . Este porcentaje 
posiblemente corresponda a estudiantes repitentes.

Estos resultados se pueden resumir en lo siguiente: los estudiantes que más asisten, obtienen las mejores calificaciones y estos, a su vez, son los que tienen mayor participación. En otras palabras "los estudiantes de mayor asistencia están mejor preparados para aprovechar las oportunidades de participación". Esto daría respuesta a la pregunta: los estudiantes de bajo rendimiento no se niegan a participar, sólo no están preparados para aprovechar las oportunidades.

Por otra parte, la distribución de la asistencia y la participación aparece en la TABLA 4.

TABLA 4. Porcentaje de asistencia y porcentaje de participación acumulada.

\begin{tabular}{|c|c|c|c|}
\hline $\begin{array}{c}\text { Participación } \\
\text { acumulada }\end{array}$ & $\begin{array}{c}\text { Porcentaje de } \\
\text { asistencia }\end{array}$ & $\begin{array}{c}\text { Participación } \\
\text { acumulada }\end{array}$ & $\begin{array}{c}\text { Porcentaje de } \\
\text { asistencia }\end{array}$ \\
\hline 0 & 26 & 14 & 95 \\
\hline 1 & 47 & 20 & 95 \\
\hline 3 & 58 & 28 & 95 \\
\hline 7 & 58 & 37 & 95 \\
\hline 7 & 68 & 48 & 95 \\
\hline 7 & 68 & 48 & 100 \\
\hline 8 & 74 & 51 & 100 \\
\hline 9 & 79 & 55 & 100 \\
\hline 11 & 79 & 60 & 100 \\
\hline 12 & 84 & 65 & 100 \\
\hline 12 & 84 & 73 & 100 \\
\hline 14 & 89 & 80 & 100 \\
\hline 14 & 95 & 90 & 100 \\
\hline 14 & 95 & & 100 \\
\hline
\end{tabular}

En el gráfico 4 se observa claramente que, aquellos que asistieron al $95 \%$ de las clases, acumularon el $86 \%$ de los puntos por participación, mientras que los que asistieron al menos del $80 \%$ de las clases, sólo obtuvieron el $11 \%$ de los puntos por participación. En el

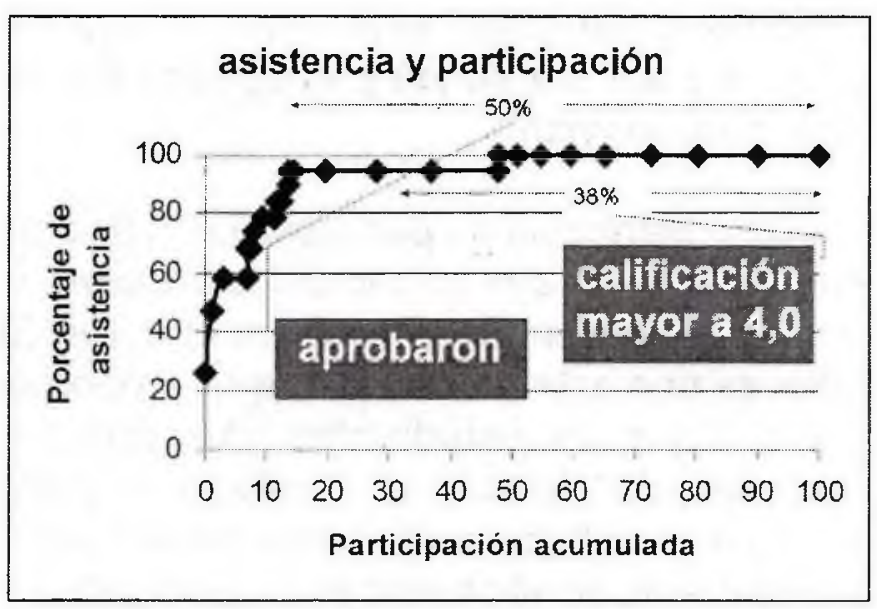

GRAFICO 4. Asistencia y participación acumulada

grupo que asistió a más del $95 \%$ de las clases un $38 \%$ la aprobó con más de 40 y $50 \%$ simplemente la aprobó. En la Tabla 1 puede observarse que en el grupo de estudiantes, cuya calificación es 45 o superior, el $70 \%$ de ellos, fueron los que más participaron, con más del $5 \%$ del total de puntos por participación; mientras que en el resto del grupo (es decir, con calificación menor a 45 ) sólo un $24 \%$ obtuvo una buena participación (superior al $5 \%$ del total de puntos de participación). Se toma una participación superior al 5\% como buena, porque corresponde al cuartil superior de los datos de participación.

En otras palabras: un cuarto de los estudiantes de calificación baja, participaron, mientras que la participación el grupo de alta calificación fue de $3 / 4$.

La estrategia de aumentar la participación tenía como fin lograr que los estudiantes de bajo rendimiento obtuvieran mejores resultados, que estuvieran más cercanos de aprobar la asignatura al final del semestre. Para ello, se tenía a mano la lista de los estudiantes ordenada según el rendimiento académico y, para que los estudiantes de bajo rendimiento pudieran aumentar su calificación, siempre se empezaba por darles la oportunidad a ellos, preguntándoles en primera instancia. Se le preguntaba a uno, si no sabia, se le preguntaba al siguiente y, luego 
del tercer intento fallido, se preguntaba en forma general y ahí era donde participaban los de mayor rendimiento!

Como la estrategia de participación individual no logró mejorar el resultado de los estudiantes de bajo rendimiento, se propuso un taller. El taller es una actividad en la que se proponen ejercicios a los estudiantes para que los resuelvan con la asesoría del profesor. El grupo se divide en sub grupos de a tres, liderados por un estudiante de alto rendimiento. Pero otra vez se presentó una sorpresa: sólo un tercio de los estudiantes que respondieron bien el taller pasaron el parcial siguiente, que tenía las mismas preguntas a resolver propuestas en el taller. Esto indicaba que las $2 / 3$ partes no participaron activamente en la solución del mismo y es una voz de alarma para los profesores que proponen trabajos para hacer en casa o peor aún, que permiten que los estudiantes resuelvan los exámenes por fuera del salón. Esta experiencia muestra que los trabajos hechos fuera de clase deben ser debidamente sustentados por los estudiantes, de otra manera, gran parte de ellos sólo participan en la firma del documento a entregar.

RESULTADOS DEL TALLER- EXAMEN

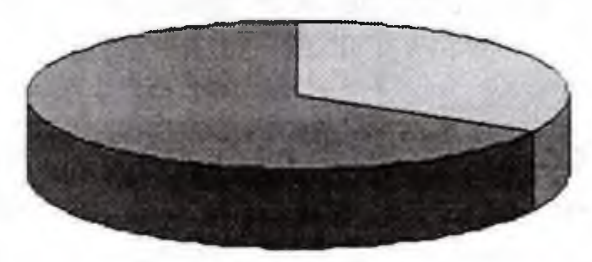

Qaprobaron ureprobaron

GRAFICO 5. Resultados del Taller - examen.

Resumiendo: el bajo rendimiento tiene relación con la poca asistencia y porque los estudiantes que no asisten no se ponen al día. La reglamentación es clara: si un estudiante no asiste al $80 \%$ de las clases, pierde por fallas.
Este estudio parece afirmar que la experiencia respalda la necesidad de esta norma.

\section{Punto de vista de los profesores del área}

Es importante comentar que los profesores que dictan esta asignatura, habian expresado las siguientes opiniones sobre el microdiseño de mecánica y electromagnetismo y sobre la forma de aumentar la calidad del proceso educativo: La intensidad horaria les parece correcta, pero no estaban de acuerdo con las prácticas de laboratorio cada quince días. El número adecuado de estudiantes en el laboratorio no debería ser superior a 18 como se estaba presentado, lo mismo el número adecuado de estudiantes por curso no debe ser mayor a 36 , Consideraron que no existe material adecuado para las prácticas de laboratorio y que son deficientes los salones y señalan como dificultad la deficiente dotación de salones multimediales. Consideraron que las unidades temáticas no deben ser cambiadas, Un $70 \%$ piensa que las estrategias de evaluación deben replantearse y el $20 \%$ afirmó no evaluar de acuerdo al microdiseño.

Al $75 \%$ le aprueban menos del $50 \%$ de los alumnos. El $100 \%$ consideró que el bajo rendimiento de sus alumnos se debe a la deficiente preparación en matemáticas, mientras un $20 \%$ considera mala la ubicación del curso en el semáforo.

Un $80 \%$ no está de acuerdo en quitar el prerequisito de física - mecànica para física electromagnética y el $60 \%$ de ellos señaló que hay necesidad de incrementar el número de cursos de física en ingeniería, para igualar a las demás universidades del pais, las cuales tienen un alto componente en ciencias básicas.

Los profesores están $100 \%$ de acuerdo en que los grupos no debieran ser grandes. La Calidad de la enseñanza no puede ser igual en la medida en que la atención y asesoría se dispersa en 
grandes grupos y no es posible atender las necesidades particulares de cada uno de los alumnos. Los cursos grandes son posibles, sólo en la medida en que se nombren varios profesores auxiliares, los cuales, dentro de la política de renovación generacional van adquiriendo las habilidades y sobre todo el conocimiento del entorno que puede brindar un profesor que está ad portas de pensionarse. Las clases magistrales funcionan en los países europeos porque los grandes grupos son divididos en pequeños grupos de 10 a 15 estudiantes bajo la tutela de un doctor que los guía en las tareas y ejercicios aplicación.

\section{Conclusiones}

Incrementar la participación de los estudiantes resultó ser un elemento muy dinamizador $y$ vivificante en las clases y, efectivamente, es un elemento que les ayuda a los mejores estudiantes a tener mayor éxito académico. Las clases resultaron muy animadas y se logra un clima de confianza muy adecuado para el proceso enseñanza - aprendizaje.

No se puede afirmar lo mismo para los estudiantes de bajo rendimiento, los cuales no mejoran su calificación.

\section{Recomendaciones}

Una forma de facilitar que los estudiantes puedan asistir y que no se atrasen es utilizar los recursos que ofrecen las nuevas tecnologias de la información y el desarrollo de la www. El estudiante puede cumplir con los compromisos académicos que señale el profesor a través de la red, asi como también, con las lecturas que debe realizar para ponerse al dia. Si no se hace uso de la red, la pregunta entonces sería: Cómo aumentar los índices de asistencia?

Otra forma es la de utilizar los mejores estudiantes como monitores, en programas formales apoyados por la universidad, tanto financieramente, pagándoles lo justo por ese

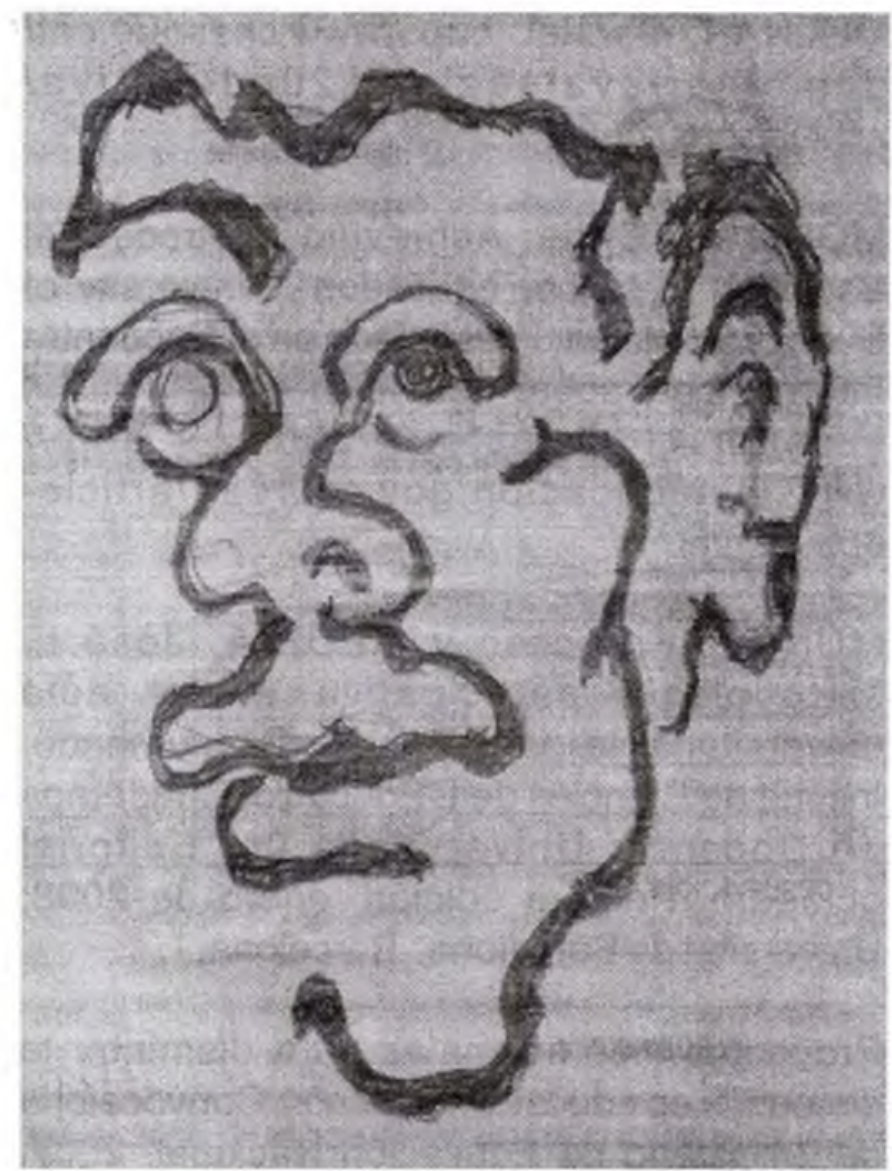

trabajo; como en recursos tecnológicos: aumentar el número de computadores por estudiantes, préstamo de portátiles con acceso a red inalámbrica, subsidiar la asistencia a salas de internet de la ciudad, de tal manera que exista mayor contacto de los estudiantes con los profesores y monitores.

\section{Bibliografía}

Acuerdo 005 de 2005 del Consejo Superior Universitario

Acuerdo 027 de 2010 del Consejo Superior Universitario

ICFES La Educación Superior en Colombia Resumen estadístico 1991-1999 s/f

MEN Boletin informativo No 7. Diciembre del 2006. 
MOLNAR, Gabriel. http://www.chasque.net/ gamolnar/evaluacion $\% 20$ educatival evaluacion.01.html

MORGAN, Chris. Achieving a succesfull transition to higher education". University of Sydney. Australia. Presentado en el Encuentro Internacional sobre deserción en Educación superior. Octubre 2004. http:// www. mineducacion.gov.co/1621/article85600.html

MUÑOZ, Francesc y MEDINA, José L. Metodología participativa en el aula universitaria. la participación del alumnado. Institut de Ciéncies de L'Educació. Cuadernos de Docencia Universitaria 04. Editorial octaedro. Primera edición: enero de 2008. Universitat de Barcelona. Barcelona.

Proyectos institucionales para disminuir la deserción en educación superior. Convocatoria del Ministerio de Educación Nacional. 2007. http://www.mineducacion.gov.co/1621/article133057.html
RAMA, Claudio. Las tendencias de la educación superior en América Latina. Feb 23, 2007

SANCHEZ, Guillermo; NAVARRO, Wisberto y GARCÍA, Alejandro D. Factores de deserción estudiantil en la Universidad Surcolombiana. Revista Paideia, mayo 2009. Universidad Surcolombiana.

Taller con profesores de física efectuado por el área de física de la Facultad de Ciencias Exactas y Naturales /09/2006.

TINTO, Vincent "El abandono en los estudios superiores: una nueva perspectiva de las causas del abandono y su tratamiento", Cuadernos de planeación universitaria, num. 2, México: UNAM. (1992).

UNESCO. Digital observatory for higher education in latin america and the caribbean. April 2005.

Universidad de la República de Uruguay. Comisión Sectorial de Enseñanza Bases del llamado a proyectos de investigación: deserción estudiantil año 2003. 